\title{
Abbreviations Used in the Book
}

AIDE Attention, Interpretation and Decision-making during Engagement

BAK Beliefs, Assumptions and Knowledge

CA Conversation Analysis

CA-for-SLA Conversation Analysis-for-Second Language Acquisition

CDST Complex Dynamic Systems Theory

CLIL Content and Language Integrated Learning

EFL English as a Foreign Language

ESL English as a Second Language

L2 Second Language

MMR Mixed-Methods Research

NNEST Non-Native English Speaking Teacher

PLLT Psychology of Language Learning and Teaching

PST Pre-Service Teacher

QUANT Quantitative

QUAL Qualitative

SCT Sociocultural Theory

SLA Second Language Acquisition

SLTE Second Language Teacher Education

TBLT Task-Based Language Teaching

TESOL Teaching English to Speakers of Other Languages

ZPD Zone of Proximal Development 